\title{
Treatment of Helicobacter pylori infection: management of patients with ulcer disease by general practitioners and gastroenterologists
}

\author{
G N J Tytgat
}

\begin{abstract}
Summary
Knowledge of the importance of Helicobacter pylori infection is still fragmentary. Currently only a minority of patients with ulcers receive adequate eradication therapy. Ideally there should be no difference in the level of knowledge between general practitioners and gastroenterologists. Yet in practice there is a substantial difference. The results obtained in highly selected clinical trials do not reflect results of practice in the real world. The gap can only be narrowed through careful mass education. The role of testing for $H$ pylori infection in primary care practice needs to be clarified and the problem of erratic treatment by general practitioners and specialists needs to be resolved. Adequate response to these problems will require the creation of "regional platforms" where both primary care physicians and specialists decide on empirical therapy, eradication strategy and referral of dyspeptic patients.
\end{abstract}

\section{Introduction}

The spread of scientific information regarding the importance of $H$ pylori infection in humans has proceeded at an unprecedented rate. Despite this explosion of information, for many physicians knowledge is still fragmentary and therefore the therapeutic consequences are incomplete, erratic and sometimes nonexistent. Theoretically there should be no difference in the level of knowledge and the therapeutic approach to $H$ pylori infection between general practitioners (GPs) and specialist gastroenterologists. Yet in practice there is regrettably a substantial difference. The gap can only be narrowed through careful education of both GPs and gastroenterologists given that new strategies are emerging almost daily. The most important question to be answered for GPs is the role of testing for $H$ pylori in the management of patients presenting with dyspeptic symptoms or even signs and symptoms of peptic ulcer disease. With the advent of simple office based tests for $H$ pylori diagnosis, further studies are warranted on the economics of $H$ pylori testing and treatment in patients with suspected but unconfirmed peptic ulcer disease.

Department of Gastroenterology and Hepatology, University of Amsterdam Academic Medical Centre, Meibergdreef 9, 1105 AZ Amsterdam Zuidoost, The Netherlands G N J Tytgat
Level of knowledge of $\boldsymbol{H}$ pylori infection Data on the level of knowledge of the role of $H$ pylori in peptic ulcer disease are still limited. ${ }^{1}$ Fendrick et al recently interviewed 1350 GPs and internists. ${ }^{2}$ Figure 1 illustrates the use of $H$ pylori eradication therapy by GPs and specialists. Gastroenterologists were more aware of the relation between $H$ pylori infection and peptic ulcer disease and adapted their practice more quickly to this emerging information.
More recently, Breuer et al interviewed a large number of American physicians. ${ }^{3}$ Table 1 summarises the salient features of that study. It is obvious from these recent data that there are still major differences in the overall appreciation of the importance of $H$ pylori infection by GPs and gastroenterologists. More recent evidence indicates that most GPs and gastroenterologists are aware of the causal relation between $H$ pylori infection and duodenal ulcer disease, but less so for gastric ulcer disease. However, application of this new information by the former is still insufficient. ${ }^{3}$

\section{How should peptic ulcer disease be diagnosed?}

A minority of patients with dyspepsia seen by GPs have ulcer disease and a genuine ulcer diathesis. Whether symptoms can be used to diagnose peptic ulcer disease accurately, as opposed to functional dyspepsia or other organic diseases, is a matter of controversy. Duodenal ulcer disease can be accurately diagnosed on the basis of symptoms in no more than $25 \%$ of cases. ${ }^{4}$ Practically, ulcer disease can only be diagnosed by endoscopy or, rarely now, by radiology. Yet not every dyspeptic patient should undergo endoscopy. Hence, how does the GP distinguish a trivial episode of dyspepsia from a significant one?

An increasing number of GPs favour the so-called test and treat strategy. Accordingly, patients under 45 years of age without alarm symptoms are being tested and $H$ pylori eradication therapy is prescribed for those with a positive result. The percentage of those with an ulcer diathesis in the overall population of dyspeptic patients is usually small. Although there are exceptions, ${ }^{5}$ the average figure is around $10 \%$. This suggests that most patients are more likely to have functional dyspepsia. However, so far there is not much evidence from randomised controlled trials suggesting that eradication of $H$ pylori is more effective than placebo in relieving dyspeptic symptoms. ${ }^{36}$ Despite a plethora of negative efficacy studies and the fact that more than half of the patients will experience recurrent dyspeptic symptoms despite being cured of their infection, many GPs are now willing to go ahead with treatment as they feel that symptoms might improve. There is indeed the occasional patient who experiences prolonged benefit after cure of $H$ pylori infection. Moreover, there is also the paucity of alternative effective treatments and this strategy will catch that unknown percentage of patients who have a genuine ulcer diathesis.

A practical consequence of this test and treat strategy is that, for many patients with ulcer 


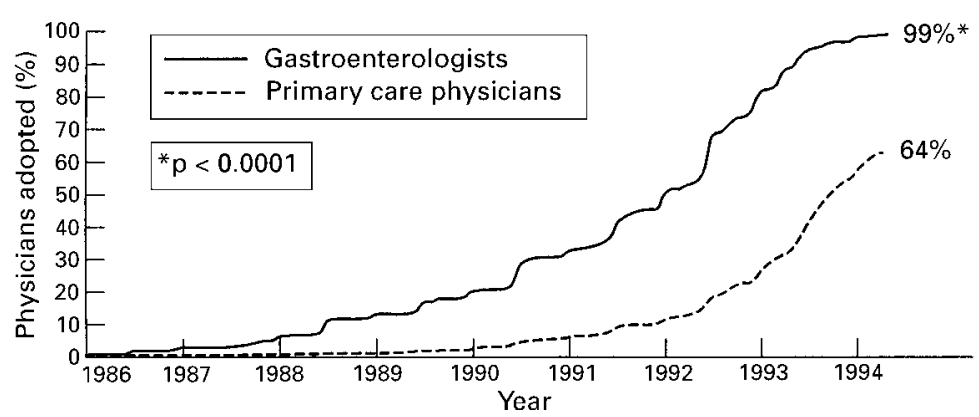

Figure 1 Adoption of $H$ pylori eradication therapy and timing of first use by specialty. Adapted from Fendrick et al. ${ }^{2}$

diathesis, no proper diagnosis will ever be reached. This would be perfectly acceptable if appropriate treatment and proper monitoring of its therapeutic efficacy were always available to GPs. This would protect treatment failures from recurrent ulceration and potential complications. Long term data to prove that such a test and treat strategy is indeed realistic and safe are currently lacking. Agréus and Talley ${ }^{8}$ recommend prompt endoscopy for patients with a positive result on $H$ pylori testing. If it is not possible/advisable to arrange a prompt endoscopy, they recommend empirical treatment of $H$ pylori instead, provided the screening test for $H$ pylori is reliable.

\section{Cost-benefit analysis}

Several cost-benefit analyses have been published $^{9-14}$ and all came to the conclusion that a test and treat strategy excluding endoscopy is the most cost-effective approach. Yet all those studies are based on the fact that the authors knew/supposed that the patient had ulcer disease. If one wants to restrict therapy only to patients with ulcer disease, then there would have to be a reduction in the cost of endoscopy to make referral for this test cost-effective. Other weaknesses of many of the cost-benefit analyses include the unknown percentage of dyspeptic patients who might improve on being cured of their infection and the fact that many features are difficult to quantify or to evaluate, such as bacterial resistance and the cost of adverse events or side effects-for example, severe diarrhoea, pseudomembranous colitis, ulcer complications after failed cure, etc. Rapidly changing therapeutic strategies also render many calculations obsolete. Finally all estimates reflect practice in a particular geographical area and cannot be extrapolated to other countries.

Table 1 Summary of the salient features of the study by Breuer et al. ${ }^{3}$ In total, 3117 physicians were interviewed

\begin{tabular}{lll}
\hline & Gastroenterologists & General practitioners \\
\hline H pylori is a causative agent in duodenal ulcer & $94 \%$ & $68 \%$ \\
H pylori is a causative agent in gastric ulcer & $72 \%$ & $68 \%$ \\
H pylori is definitely related to gastric cancer & $21 \%$ & $9 \%$ \\
H pylori is causally related to MALT lymphoma & $55 \%$ & $5 \%$ \\
Use of ineffective, unproven regimens & $11 \%$ & $31 \%$ \\
$\quad$ (103 regimens in use) & & \\
\hline
\end{tabular}

MALT, mucosa associated lymphoid tissue.
Treatment practice in the real world

Highly effective eradication strategies are available and comprise a proton pump inhibitor or ranitidine bismuth citrate with two antimicrobials or quadruple therapy (proton pump inhibitor plus bismuth plus tetracycline plus metronidazole). Although these triple/ quadruple drug therapies give excellent results in clinical trials, their efficacy in practice will almost certainly be less. Indeed, the results in highly selected clinical trials do not reflect results of practice in the real world. Penston and Mistry ${ }^{15}$ recently evaluated five general practices in Scotland. Fifty six different regimens were used in 154 patients. Two thirds of the patients were not tested for $H$ pylori infection before receiving eradication therapy. Half the patients received dual therapy which has now been superseded, $52 \%$ complained of symptom recurrence and $47 \%$ required further treatment for their symptoms. The results of a recent American study are equally disturbing. One hundred and three different regimens were used and ineffective regimens were prescribed by $31 \%$ of the GPs compared with $11 \%$ of the gastroenterologists. ${ }^{3}$

These appalling results should not automatically lead to the conclusion that appropriate treatment by GPs is impossible. Some recent studies have shown equally high cure rates can be achieved by both GPs and specialists when appropriate eradication strategies, even quadruple therapy, are used (Lai et al, manuscript submitted). ${ }^{16}$ However, it is fair to conclude that we need more comprehensive data on how GPs and specialists, outside the context of a clinical trial, are treating suspected $H$ pylori infection. Choice of treatment differs widely depending on the country and also within a particular geographical area. New and more effective therapies, particularly monotherapies, which are easy to take and of short duration, are urgently needed. Until these are available, the only possible way to reduce the chaos and confusion is to create "regional platforms" where both GPs and specialists decide together which treatments to use and how to monitor their efficacy.

\section{Do patients with ulcer disease receive appropriate treatment?}

It has now been shown by many studies that cure of $H$ pylori infection is equivalent to cure of $H$ pylori associated ulcer disease ${ }^{17}{ }^{18}$ and that recurrent infection is exceedingly rare. ${ }^{19}$ Despite these encouraging data, in reality only a minority of patients with ulcers receive appropriate antimicrobial therapy. According to Penston and Pounder ${ }^{19}$ only $9 \%$ of the subjects with peptic ulcer disease have received treatment for $\mathrm{H}$ pylori eradication. These data were derived from more than 150 sampling points in England, Scotland and Wales in 1994. According to Bodger et al only $30 \%$ of patients with previously documented peptic ulcer disease received $H$ pylori eradication therapy. ${ }^{20}$ This study took place in GP practices in the Leeds area in 1995.

Vreeburg et al analysed patients presenting with acute upper intestinal bleeding in the greater Amsterdam area (fig 2). ${ }^{21}$ Only a small 
951 Bleeding patients

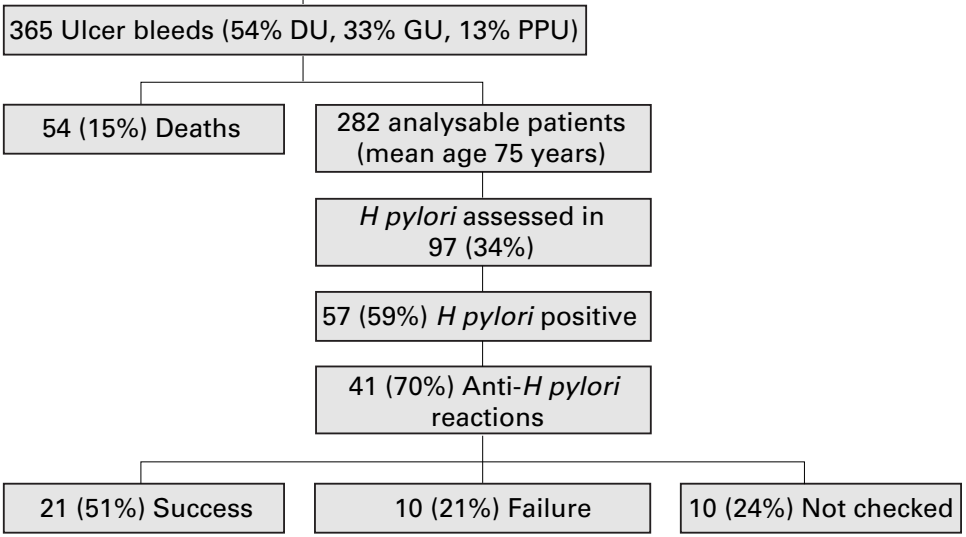

Figure 2 Upper intestinal bleeding. Amsterdam study (1994) including two academic teaching hospitals and 10 regional hospitals (catchment population 1.6 million). DU, duodenal ulcer; GU, gastric ulcer; PPU, prepyloric ulcer.

\section{Reasons for referral to specialist gastroenterologists}

- H pylori associated refractory ulceration

- Confirmation of diagnosis

- Microbial resistance pattern

- Primary $H$ pylori negative ulceration

- Recurrent ulceration despite eradication of $H$ pylori

- Idiopathic acid/pepsin hypersecretion

- Overt/covert use of aspirin or nonsteroidal anti-inflammatory drugs

- Crohn's disease

- Gastrinoma

- Refractory/complicated ulcer disease

fraction of patients with $H$ pylori associated bleeding ulcers ultimately received suitable eradication therapy.

\section{Conclusion}

Some experts have expressed the view that $H$ pylori infection is simply an eradicable infectious disease which should be eliminated in all those infected. This is quite a shocking idea to many of us, yet this approach needs to be seriously considered by health care providers. In reality we are still far away from this idealised situation.

Without doubt GPs will play a key role in solving the H pylori "problem" because they are the ones who see the patients first. Solving the problem will require optimal teaching and education of both GPs and specialists and the creation of regional platforms where the overall approach and the selection of treatment will be decided upon depending on the local antimicrobial resistance patterns.

Although GPs will increasingly treat most of those patients with $H$ pylori associated ulcer diathesis, some patients will need to be referred for specialist investigation (summarised in the box). Discovering the cause of ulceration in those patients will undoubtably require expert endoscopy with multiple biopsies and occasionally gastric secretory and hormonal analysis.

It is probably wise to discourage the use of newer antimicrobial regimens until their effi- cacy has been established by rigorous studies. This certainly would help to reduce confusion among clinicians. Primary care physicians should concentrate on a few effective regimens which have been approved by the regional platforms. Regular review by the regional platform is obviously essential and includes monitoring of microbial sensitivity and drug availability. Effective education of both GPs and specialists with regard to which treatment to use and when is urgently needed. Further approval of these regimens by the national health authorities may well be the best way to ensure overall good management and compliance.

Several unresolved questions and issues continue to cloud current treatment of $H$ pylori infection, including how $H$ pylori positive patients with dyspepsia should be treated, how to tackle the link between infection with $H$ pylori and gastric cancer in a cost-effective way, and how to encourage physicians to use more effective treatment regimens. The agenda of issues to be resolved remains impressive.

1 Malfertheiner P, Breuer T. What is the role of the primary care physician in the treatment of Helicobacter pylori infection? In: Hunt RJ, Tytgat GNJ, eds. Helicobacter pylori. Basic mechanisms to clinical cure. Dordrecht: Kluwer Academic Publishers, 1996:366-73.

2 Fendrick AM, Hirth RA, Chernew ME. Differences between generalist and specialist physicians regarding Helicobacter pylori and peptic ulcer disease. Am $\mathcal{f}$ Gastroenterol 1996;91:1544-8.

3 Breuer T, Goodman K, Malaty HM, et al. How do clinicians practicing in the US manage Helicobacter pylori-related practicing in the US manage Helicobacter pylor

4 DeLuca VA Jr, Winnan GG, Sheahan DG, et al. Is gastroduodenitis part of the spectrum of peptic ulcer disease? F Clin Gastroenterol 1981;3(suppl 2):17-22.

5 McColl KEL, El-Nujumi A, Murray L, et al. The Helicobacter pylori breath test: a surrogate marker for peptic ulcer disease in dyspeptic patients. Gut 1997;40:302-6.

6 Tytgat GNJ. Role of Helicobacter pylori in chronic dyspepsia. Dordrecht: Kluwer Academic Publishers, 1998.

7 Jones R. Non-ulcer dyspepsia: great expectations? Am $\mathcal{J}$ Jastroenterol 1996;91:628-9.
Jones R. Non-ulcer dyspepsias

8 Agréus L, Talley N. Challenges in managing dyspepsia in Agreus L, Talley N. Challenges in mana
general practice. $B M f$ 1997;315:1284-8.

9 Fendrick AM, Chernew MI, Hirth RA, et al. Alternative management strategies for patients with suspected peptic ulcer disease. Ann Intern Med 1995;123:260-8.

10 Sonnenberg A, Townsend W. Costs of duodenal ulcer therapy with antibiotics. Arch Intern Med 1995;155:928-9.

11 Silverstein FE, Petterson T, Talley NJ. Initial endoscopy or empirical therapy with or without testing for $\mathrm{H}$. pylori for dyspepsia: a decision analysis. Gastroenterology 1996;110: 302-6.

12 Vakil N, Fennerty MB. Cost-effectiveness of treatment regimens for the eradication of Helicobacter pylori in duodenal mens for the eradication of Helicobacter pylor

13 Taylor JL, Zagari M, Murphy K, et al. Pharmacoeconomic comparison of treatments for the eradication of Helicobacter pylori. Arch Intern Med 1997;157:87-97.

14 O'Brien B, Goeree R, Hunt RW, et al. Cost effectiveness of alternative Helicobacter pylori eradication strategies in the management of duodenal ulcer. Can $\mathcal{f}$ Gastroenterol 1997;11:323-31.

15 Penston JG, Mistry KR. Eradication of Helicobacter pylori in general practice. Aliment Pharmacol Ther 1996;10:13945 .

16 Vautier G, Scott BB. A one-week quadruple eradication regimen for Helicobacter pylori in routine clinical practice. Aliment Pharmacol Ther 1997;11:107-8.

17 Van der Hulst RWM, Rauws EAJ, Köycü B, et al. Prevention of ulcer recurrence after eradication of Helicobacter pylori: a prospective long-term follow-up study. Gastroenterology 1997;113:1082-6.

18 Van der Hulst RWM, Rauws EAJ, Köycü B, et al. H. pylori reinfection is virtually absent after successful eradication. $\mathcal{F}$ Infect Dis 1997;176:196-200.

19 Penston JG, Pounder RE. A survey of dyspepsia in Great Britain. Aliment Pharmacol Ther 1996;10:83-9.

20 Bodger K, Daly MJ, Heatley RV. Prescribing patterns for dyspepsia in primary care: a prospective study of selected general practitioners. Aliment Pharmacol Ther 1996;10: 889-95.

21 Vreeburg EM, Snel P, De Bruijne JW, et al. Acute upper gastrointestinal bleeding in the Amsterdam area: incidence, diagnosis, and clincial outcome. Am $\mathcal{f}$ Gastroenterol 1997;92:236-43. 